\title{
Neutral Current Coupling Constants from Neutrino- and Antineutrino-Electron Scattering
}

\author{
CHARM II Collaboration
}

P. Vilain ${ }^{1}$, G. Wilquet ${ }^{1}$

Inter-University Institute for High Energies (ULB-VUB), Brussels, Belgium

W. Flegel, H. Grote, T. Mouthuy ${ }^{2}$, H. Øverås, J. Panman, A. Rozanov ${ }^{3}$, K. Winter, G. Zacek, V. Zacek

CERN, Geneva, Switzerland

R. Beyer ${ }^{4}$, F.W. Büsser, C. Foos, L. Gerland T. Layda, F. Niebergall, G. Rädel,

P. Stähelin, T. Voss

II. Institut für Experimentalphysik ${ }^{5}$, Universität Hamburg, Hamburg, Germany

D. Favart, G. Grégoire, E. Knoops ${ }^{6}$, V. Lemaître

Université Catholique de Louvain, Louvain-la-Neuve, Belgium

P. Gorbunov, E. Grigoriev, V. Khovansky, A. Maslennikov

Institute for Theoretical and Experimental Physics, Moscow, Russian Federation

W. Lippich, A. Nathaniel, A. Staude, J. Vogt

Sektion Physik ${ }^{5}$ der Universität München, Munich, Germany

M. Caria ${ }^{7}$, A.G. Cocco, B. Eckart ${ }^{8}$, A. Ereditato, G. Fiorillo, V. Palladino, P. Strolin Università e Istituto Nazionale di Fisica Nucleare (INFN), Naples, Italy

A. Capone, D. De Pedis, E. Di Capua ${ }^{9}$, U. Dore, A. Frenkel-Rambaldi, P.F. Loverre, D. Macina, G. Piredda, R. Santacesaria

Università 'La Sapienza' e Istituto Nazionale di Fisica Nucleare (INFN), Rome, Italy

(submitted to Phys. Lett. B)

Abstract

We are reporting on a determination of neutral current coupling constants from a study of differential cross sections of (anti)muon-neutrino electron scattering. The results were obtained with the CHARM-II detector which was exposed to the CERN wide band neutrino beam in the years 1987 to 1990 . A total of about $2100 \nu e$ and $2200 \bar{\nu} e$ scattering events were observed. From a comparison between the data and predicted event distributions, the effective vector and axial-vector neutral current coupling constants of the electron were determined to be $g_{V}^{e}=-0.025 \pm 0.019$ and $g_{A}^{e}=-0.503 \pm 0.018$.

1 National Foundation for Scientific Research, Belgium

2 now at Centre de Physique des Particules de Marseille, Faculté de Luminy, Marseille, France

3 on leave of absence from ITEP. Moscow, Russian Federation .

4 now at CERN, Geneva, Switzerland.

5 supported by the German Bundesministerium für Forschung und Technologie (BMFT), under contract number 05-4HH22P and $05-4 \mathrm{MU} 12 \mathrm{P}$.

6 Inter-University Institute for Nuclear Science, Belgium

7 now at University of Perugia, Italy.

8 now at University of Basel, Switzerland.

${ }^{9}$ Dipartimento di Fisica, Università di Ferrara, Italy 



\section{Introduction}

Very precise measurements of electroweak parameters have been made at the electron-positron collider LEP at CERN[1]. In view of higher order corrections, however, it remains of great interest to compare these measurements at the $Z^{0}$-resonance with those performed at lower energy where processes occur by virtual $Z^{0}$ exchange. Neutrinoelectron scattering is perfectly suited for such a comparison, since the process is purely leptonic and, in terms of coupling constants, nearly equivalent to the annihilation of electrons and positrons into lepton pairs.In a previous publication [2], we obtained a result for the electroweak mixing angle, $\sin ^{2} \theta_{W}$, derived from a measurement of the cross section ratio $R=\sigma\left(\nu_{\mu} e\right) / \sigma\left(\bar{\nu}_{\mu} e\right)$. We present here a new, improved analysis based on a measurement of the differential cross sections of muon-neutrino and muon-antineutrino scattering off electrons [3]

$$
\begin{aligned}
\frac{\mathrm{d} \sigma_{\bar{\nu}}^{\nu}=}{\mathrm{d} y} & \frac{G_{F}^{2} m_{e}}{2 \pi} E_{\nu} \times \\
& {\left[\left(g_{V} \pm g_{A}\right)^{2}+\left(g_{V} \mp g_{A}\right)^{2}(1-y)^{2}\right] }
\end{aligned}
$$

where $y=\left(1-\cos \theta^{*}\right) / 2$ and $\theta^{*}$ is the scattering angle in the c.m. system, $g_{V}$ and $g_{A}$ the vector and axial-vector coupling constants of the electron to the neutral current respectively. In addition we include the knowledge on absolute $\nu$-fluxes and selection efficiencies. This new method enables us to determine, simultaneously two electroweak parameters and increases the experimental sensitivity because of the use of energy distributions of the events.

The CHARM II detector was built to study neutrino-electron scattering [4]. It consists of a massive target calorimeter followed by a muon spectrometer. The calorimeter is instrumented with streamer tubes equipped with digital and analog readout to measure the energy and the direction of particles produced. The detector was exposed to the horn focused wide band neutrino beam (WBB) at CERN. Neutrinos were produced by a $450 \mathrm{GeV}$ proton beam accelerated in the Super Proton Synchrotron (SPS).

The signature of neutrino-electron scattering is a single, forward scattered electron producing an electromagnetic shower in the calorimeter. The variable $E_{e} \theta_{e}^{2}$, the product of electron energy and the square of the scattering angle, is kinematically constrained to values smaller than $1 \mathrm{MeV}$. This fact is used to separate $\nu e$-scattering from the background of semileptonic events which has a broad distribution.

\section{Analysis method}

The differential cross section as it is given in eq. (1) is valid only for muon(anti)-neutrinos. Due to the contamination of neutrinos of opposite helicity and electron-neutrinos $\left(\nu_{e}\right.$ and $\left.\bar{\nu}_{e}\right)$ in the neutrino beam, the measured differential cross sections contain contributions from four different neutrinoelectron scattering processes. Since the four differential cross sections depend on the same electroweak parameters, the measurements in the $\nu$ and $\bar{\nu}$-beam ${ }^{1}$ lead to a simultaneous measurement of two electroweak parameters as will be shown below.

The general form of the neutrino-electron scattering cross section can be written as

$$
\frac{\mathrm{d} \sigma}{\mathrm{d} y}=\frac{G_{F}^{2} m_{e}}{2 \pi} E_{\nu} \sum_{i=1}^{3} A_{i} g_{i}
$$

where the $g_{i}$ are different combinations of electroweak coupling constants:

$$
\begin{aligned}
& g_{1}=\left(g_{V}+g_{A}\right)^{2} \\
& g_{2}=\left(g_{V}-g_{A}\right)^{2} \\
& g_{3}=\left(2+g_{V}+g_{A}\right)^{2}
\end{aligned}
$$

The expressions $A_{i}$ are given in table 1 for the four processes involved. The third term in (2) accounts

Table 1: Terms of the differential cross section for different processes.

$$
\begin{array}{llll}
\text { Process } & A_{1} & A_{2} & A_{3} \\
\nu_{\mu} e \rightarrow \nu_{\mu} e & 1 & (1-y)^{2} & 0 \\
\bar{\nu}_{\mu} e \rightarrow \bar{\nu}_{\mu} e & (1-y)^{2} & 1 & 0 \\
\nu_{e} e \rightarrow \nu_{e} e & 0 & (1-y)^{2} & 1 \\
\bar{\nu}_{e} e \rightarrow \bar{\nu}_{e} e & 0 & 1 & (1-y)^{2}
\end{array}
$$

for the interference of neutral and charged currents in electron-neutrino electron scattering.

${ }^{1}$ We call the beam produced by focussing positive parents the $\nu$-beam and by negative parents the $\bar{\nu}$-beam. 
The four differential cross sections (2) depend on two parameters only, either on $g_{A}$ and $g_{V}$, or using the Standard Model relation between the coupling constants and the electroweak mixing angle:

$$
g_{V}^{e}=\rho\left(-1 / 2+2 \sin ^{2} \theta_{W}\right) \text { and } g_{A}^{e}=-\rho / 2,
$$

on $\sin ^{2} \theta_{W}$ and $\rho$, the relative coupling strength of the neutral current with respect to the charged current.

The measured event rate, e.g., in the neutrino beam is given by

$$
\mathrm{d} n^{\nu e} / \mathrm{d} y=\sum_{i=1}^{3} f_{i}^{\nu e} g_{i}
$$

where the differential distributions $f_{i}^{\nu e}$ contain all information about the target density, the neutrino flux and energy spectra, the cross section expressions $A_{i}$, and the experimental resolutions and acceptances. All these quantities are either known by calculation or measured as described below.

Electroweak parameters are obtained from a simultaneous fit of modelled differential distributions $f_{i}^{\nu e}$ to the data collected in the $\nu$ - and $\bar{\nu}$-beam. In this way a direct determination of the coupling constants is possible. Previous $\nu e$-experiments have also deduced the coupling constants from $\nu_{\mu} e$ and $\bar{\nu}_{\mu} e$ cross sections $[5,6]$ but with much lower statistics.

\section{Data analysis}

The data presented here were collected during the years $1987-1990$ and represent $\approx 80 \%$ of the final statistics. In total $2.1 \cdot 10^{19}$ protons were delivered to the target. The data-taking in neutrino and antineutrino mode was alternated every 2-3 days to equalize the detection efficiency and protons were shared in such a way that the number of neutrinoelectron and antineutrino-electron scattering candidates was nearly equal.

\section{Neutrino flux and energy spectra}

The neutrino flux was obtained from a monitor reaction with known cross section, namely inclusive neutrino-nucleon scattering. The analysis of these reactions was based on two different methods.

For the first method neutrino-induced events were selected without separation into charged current (CC) and neutral current (NC) with an energy threshold of $3 \mathrm{GeV}$ (so-called minimum bias events). Based on the known $\mathrm{CC}$ cross section and $\mathrm{NC} / \mathrm{CC}$ cross section ratio the number of these events was converted into an integrated neutrino flux. The second method is based on CC interactions in a restricted fiducial volume in the neutrino energy range $15-60 \mathrm{GeV}$ where the CC cross section is well known. Good agreement of observed and simulated differential distributions ensured that acceptance corrections were correctly calculated. The neutrino flux was then scaled to the full energy range.

The results of the two methods show good agreement. The acceptance corrections and uncertainties in the beam composition are different for the two samples. Thus the systematic error was be reduced. The absolute flux in the $\bar{\nu}$ - beam was not determined in the same way, since the $\mathrm{CC}$ cross section in not so well known for $\bar{\nu}_{\mu}$. Instead it was determined from a measurement of the flux ratio $f=\Phi_{\bar{\nu}} / \Phi_{\nu}$ in the neutrino and antineutrino beam which had already been evaluated for the measurement of the ratio of $\nu_{\mu} e$ and $\bar{\nu}_{\mu} e$ cross sections [2].

A total energy weighted flux of main component neutrinos in the fiducial volume of the detector of $(1.68 \pm 0.08) \cdot 10^{18} \mathrm{GeV}$ and $(1.68 \pm 0.09) \cdot 10^{18} \mathrm{GeV}$ was obtained for the $\nu$ - and $\bar{\nu}$-beam, respectively. The total uncertainty on the flux measurement was found to be $4.7 \%$ and $5.2 \%$ for the neutrino and antineutrino beam, respectively.

Table 2: Neutrino beam composition.

$\begin{array}{cccc}\text { Beam Component } & \begin{array}{c}\text { Relative } \\ \text { abundance }\end{array} & \begin{array}{c}\left\langle E_{\nu}\right\rangle \\ (\mathrm{GeV})\end{array} \\ \nu & \nu_{\mu} & 1 & 23.7 \pm 0.3 \\ & \bar{\nu}_{\mu} & 0.072 \pm 0.004 & 19.2 \pm 0.5 \\ & \nu_{e} & 0.0087 \pm 0.0013 & 44.0 \pm 2.2 \\ \bar{\nu} & \bar{\nu}_{e} & 0.0017 \pm 0.0003 & 33.8 \pm 1.7 \\ & \nu_{\mu} & 0.136 \pm 0.007 & 26.3 \pm 0.5 \\ & \bar{\nu}_{\mu} & 1 & 19.1 \pm 0.2 \\ & \nu_{e} & 0.0071 \pm 0.0011 & 36.5 \pm 1.8 \\ & \bar{\nu}_{e} & 0.0043 \pm 0.0006 & 37.0 \pm 1.9\end{array}$

For the flux normalisation as well as for the modelling of the $\nu e$ event distributions the knowledge of the neutrino beam properties is essential. 
The neutrino beam spectra and the relative flux of opposite helicity components were obtained from a new analysis of charged current events with low momentum transfer. Acceptance and resolution effects were unfolded. The electron-neutrino components were determined by Monte-Carlo methods. The results on the beam composition are summarized in table 2 .

\section{Neutrino-electron scattering event selection}

The selection of neutrino-electron scattering candidates was described previously [2]. The selection efficiencies which have to be known for this analysis were determined using test beam data and found to be independent of the shower energy. A careful check of possible systematic effects due to detector instabilities was performed. The overall uncertainty of the selection efficiency of neutrino-electron scattering candidates was found to be $\pm 3.4 \%$.

The resolution functions for the energy and direction determination were obtained from a calibration of the detector in a test beam [7]. We determined the energy of electromagnetic showers from the number of streamer tube hits in the event. The relative resolution was found to be

$$
\Delta E_{e} / E_{e}=0.09 / \sqrt{ } E_{e} / \mathrm{GeV}+0.11
$$

The absolute energy scale uncertainty is estimated to be $5 \%$. For the angular resolution

$$
\Delta \theta_{e}^{\mathrm{proj}}=\left[\frac{27\left(E_{e} / \mathrm{GeV}\right)^{-2}+14}{\sqrt{ } E_{e} / \mathrm{GeV}}+1\right] \mathrm{mrad}
$$

was found. ${ }^{2}$

\section{Background determination}

The background consists of semileptonic neutrino reactions producing predominantly electromagnetic final states. In total four processes are expected to contribute to the background. The main contribution is coming from coherent ( $\left.\pi^{0} \mathrm{coh}\right)$ and diffractive ( $\pi^{0}$ dif) neutrino production of single neutral pions in $\mathrm{NC}$ interactions. Electromagnetic showers are also produced in quasi-elastic neutrinonucleon reactions of electron-neutrinos ( $\nu_{e}$ q.e.) $\mathrm{A}$

\footnotetext{
${ }^{2}$ This is equivalent to $\Delta \theta_{e}^{\mathrm{proj}} \approx 17 \mathrm{mrad} / \sqrt{ } E_{e} / \mathrm{GeV}$ in the energy range of our analysis.
}

small fraction of the background is due to inclusive neutrino reactions (incl.) with a large electromagnetic component in the final state. The background distributions $f_{i}^{B G}$ are modelled using data and Monte-Carlo techniques as described in ref. [2].

\section{Results}

\section{The fit procedure}

The experimental data and the theoretical predictions are described as double differential distributions in the kinematic variables $E_{e}$ and $E_{e} \theta_{e}^{2}$ :

$$
f=\frac{\mathrm{d}^{2} n}{\mathrm{~d} E_{e} \mathrm{~d}\left(E_{e} \theta_{e}^{2}\right)}
$$

They discriminate between signal and background in the variable $E_{e} \theta_{e}^{2}$, and determine the background composition because of their different energy $\left(E_{e}\right)$ distributions (see figure 1).

The task of the final analysis step is to fit the predicted distributions $p$ of the neutrino-electron scattering signal and the background processes

$$
p=\sum_{i=1}^{3} f_{i}^{\nu e} g_{i}+\sum_{i=1}^{4} f_{i}^{B G} b_{i}
$$

to the experimental distributions $d=f^{\text {data }}$. The coefficients $b_{i}$ determine the contributions of the four background processes.

The theoretical prediction for neutrino-electron scattering was corrected for higher order QED effects [10]. The coupling constants determined from this fit are therefore effective values including, but not corrected for, higher order electroweak effects.

The fit was performed using distributions with non-equidistant binning according to the experimental resolutions. The energy range from 3 to $24 \mathrm{GeV}$ was subdivided in 22 bins, and the $E \theta^{2}$ range from 0 to $72 \mathrm{MeV}$ in 16 bins, leading to 352 bins in the 2-dimensional distributions. The fit was performed to both neutrino and anti-neutrino data simultaneously, hence to 704 bins.

The coupling constants $g_{V}$ and $g_{A}$ and the background composition $b_{i}$ were taken as free parameters. With the ratio of diffractive to coherent pion production $\left(b_{2} / b_{1}\right)$ constrained to be equal in neutrino and antineutrino beam data [9] this adds up to nine free parameters. The result of the best fit ( $\chi^{2}=703$ for 695 d.o.f.) is illustrated in figure 1 and summarized in table 3 . From the fit results the 
number of $\nu e$ scattering events can be extracted. A total of

$$
2105 \pm 69 \text { and } \quad 2215 \pm 76
$$

events are present in the $\nu$ and $\bar{\nu}$ data sample, respectively.

The fourfold ambiguity in the determination of $g_{V}$ and $g_{A}$ which is expected from the quadratic dependence of the cross sections on the couplings, is reduced to a two-fold one owing to the presence of $\nu_{e}$ and $\bar{\nu}_{e}$ components in the beam. About $10 \%$ of the $\nu e$ events are induced by electronneutrinos, which select two solutions. Results from $e^{+} e^{-} \rightarrow e^{+} e^{-}$experiments [8] resolve the remaining ambiguity.

Table 3: Results of the fit to neutrino and antineutrino data. The errors are statistical. The coefficients $b_{i}$ are the number of background events in the full fit range.

$\begin{array}{lcc}\text { fit parameter } & \nu \text {-beam } & \bar{\nu} \text {-beam } \\ g_{V} & -0.0254 \pm 0.0138 \\ g_{A} & -0.5027 \pm 0.0069 \\ b_{1}\left(\pi^{0} \text { coh }\right) & 22238 \pm 1335 & 25723 \pm 1542 \\ b_{2} / b_{1} & 0.34 \pm 0.06 \\ b_{3}\left(\nu_{e} \text { q.e. }\right) & 4234 \pm 233 & 7413 \pm 269 \\ \left.b_{4} \text { (incl. }\right) & 681 \pm_{358}^{194} & 381 \pm{ }_{380}^{224}\end{array}$

The systematic errors are dominated by uncertainties of the background determination, of the neutrino flux measurement and of the selection efficiency. A break down of the error sources which contribute to the uncertainties in the determination of the electroweak couplings is shown in table 4.

\section{Discussion}

The results for the effective vector and axial-vector coupling constants from neutrino-electron scattering are

$$
\begin{aligned}
& g_{V}^{e}(\nu e)=-0.025 \pm 0.019 \\
& g_{A}^{e}(\nu e)=-0.503 \pm 0.018
\end{aligned}
$$

where the statistical and systematic errors have been combined in quadrature.

This result can be compared with the measurements at LEP. The $Z^{0}$ exchange diagram for $\nu e$-scattering is related by crossing symmetry to the annihilation process $e^{+} e^{-} \rightarrow e^{+} e^{-}$via $Z^{0}$ exchange. However, the two measurements refer to different $Q^{2}$ scales. Differences of the two couplings at the two scales $\left(Q^{2}=0.01 \mathrm{GeV}^{2}\right.$ and $Q^{2}=m_{Z}^{2}$ ) are expected to arise from the running of the fine structure constant $\alpha$ and the effect of the neutrino charge radius. However, these different contributions cancel almost completely, resulting in a difference of $g_{V}^{e}(\nu e)-g_{V}^{e}(L E P)=-0.002$ while individual contributions are larger by an order of magnitude. ${ }^{3}$

Table 4: Systematic errors.

$\begin{array}{lll}\text { error source } & \delta g_{V} & \delta g_{A} \\ \text { neutrino flux } & 0.006 & 0.009 \\ \text { selection efficiency } & 0.002 & 0.009 \\ \text { exp. resolutions } & 0.001 & 0.001 \\ \text { energy scale } & 0.002 & 0.004 \\ \text { background } & 0.011 & 0.009 \\ \text { beam spectra and composition } & 0.005 & 0.002 \\ \text { systematic error } & 0.014 & 0.016 \\ \text { statistical error } & 0.014 & 0.007 \\ \text { total } & 0.019 & 0.018\end{array}$

Thus it is possible to compare our result directly with those obtained from a measurement of the partial width $\Gamma_{e e}$ at the $Z^{0}$ resonance and the forward-backward asymmetry $A^{F B}$ at LEP. Figure 2 shows that results from neutrino-electron scattering have reached comparable precision in $g_{V}^{e}$. The agreement of the measurement performed at $Q^{2} \approx 0.01 \mathrm{GeV}^{2}$ with those performed at $Q^{2}=m_{Z}^{2}$ is remarkable.

Using the parametrisation (4) for the coefficients $g_{i}$ in (2) it is also possible to perform a fit to the data using the electroweak mixing angle $\sin ^{2} \theta_{W}$ and the relative coupling strength $\rho$ of neutral and charged currents as free parameters:

$$
\begin{aligned}
\sin ^{2} \theta_{\nu e} & =0.237 \pm 0.007_{\text {stat. }} \pm 0.007_{\text {syst. }} \\
\rho_{\nu e} & =1.006 \pm 0.014_{\text {stat. }} \pm 0.033_{\text {syst. }} .
\end{aligned}
$$

Using the MS renormalization scheme [11] we

${ }^{3}$ The difference depends on the choice of the top and Higgs masses. In calculations done with the program NUFITTER [10] the masses were fixed to $m_{t}=150 \mathrm{GeV}$, $m_{H}=100 \mathrm{GeV}$. 
can correct our result for higher order electroweak effects. This renormalization scheme is advantageous, because the dependence of this correction on unknown quantities, like $m_{t}$ and $m_{H}$, is rather small. Applying these corrections [10], we derived at $Q^{2}=m_{Z}^{2}$

$$
\begin{aligned}
\sin ^{2} \bar{\theta}(\nu e) & =0.237 \pm 0.010_{\text {exp. }} \pm 0.002_{\text {theor. }} \\
\bar{\rho}(\nu e) & =1.001 \pm 0.038_{\text {exp. }} \pm 0.004_{\text {theor }}
\end{aligned}
$$

where the theoretical error accounts for varying the top quark mass in the range $m_{t}=$ [80..180] $\mathrm{GeV}$ and the Higgs boson mass in the range $m_{H}=$ [50..1000] GeV. This result can be directly compared to the predictions of the Minimal Standard Model using as input parameters $\alpha, G_{F}$ and the mass measurements of the $Z^{0}$ boson performed at LEP [1]:

$$
\begin{aligned}
\sin ^{2} \bar{\theta}\left(\alpha, G_{F}, m_{Z}\right) & =0.233 \pm 0.002_{\text {theor. }} \\
\bar{\rho}\left(\alpha, G_{F}, m_{Z}\right) & =1.001 \pm 0.004_{\text {theor. }}
\end{aligned}
$$

with the theoretical errors accounting for the same sources as above. The experimental error from the uncertainty of the $Z^{0}$ mass measurement is comparably negligable.

Since the results in terms of $\sin ^{2} \theta$ and $\rho$ are practically uncorrelated we can compare our new result with that previously obtained for $\sin ^{2} \theta$ from the ratio of cross sections [2]. The systematic error due to the absolute neutrino flux and part of the error due to the background subtraction do not influence $\sin ^{2} \theta$ and cancel in the same way for the 2-parameter fit as for the cross section ratio. In fact, fixing $\rho$ to unity and leaving the absolute normalization as a free parameter in the fit, the method described above is equivalent to a measurement of the cross section ratio. Using the same data sample (taken in 1987-89) as in [2] we obtain $\sin ^{2} \theta^{\nu e}=0.236 \pm 0.008 \pm 0.007$, in good agreement with our previous publication. The small difference in the value of $\sin ^{2} \theta^{\nu e}$ arises mainly from a more accurate new calculation of the beam spectra ( $\mathrm{cf}$. table 2). The statistical error has been improved by including the information of the differential cross section in the determination of $\sin ^{2} \theta$.

In conclusion, electroweak parameters determined from the differential cross sections of neutrino-electron scattering are in very good agreement with those from LEP experiments. The ob- served agreement of measurements spanning a factor $10^{6}$ in $Q^{2}$ is a remarkable confirmation of the Standard Model. In future, more data will be included in the analysis and the analysis will be further improved.

\section{Acknowledgements}

We gratefully acknowledge the skill and dedication of our many technical collaborators who have contributed to the realization and the operation of the detector and of its associated systems. The experiment has been made possible by grants from the Inter-University Institute for Nuclear Sciences (Belgium), CERN (Geneva, Switzerland), the Bundesministerium für Forschung und Technologie (FRG), the Institute of Theoretical and Experimental Physics (Moscow, Russian Federation), and the Istituto Nazionale di Fisica Nucleare (Italy); we gratefully acknowledge their support. We should like to thank the neutrino beam staff for their competent assistance ensuring the excellent performance of their facility. The accumulation of a large data sample in this experiment has been made possible thanks to the skilful crew operating the CERN Super Proton Synchrotron. We are indebted to D.Yu. Bardin and G. Altarelli for discussions on radiative corrections.

\section{References}

[1] J. Carter, Proceedings of 1991 joined LeptonPhoton and High Energy Physics Conference, Geneva.

[2] CHARM II Collab., D. Geiregat et al., Phys. Lett. B 259 (1991) 499.

[3] G. t'Hooft, Phys. Lett. B 37 (1971) 195.

[4] CHARM II Collab., K. de Winter et al., Nucl. Inst. \& Meth. A 278 (1989) 670.

[5] CHARM Collab., J. Dorenbosch et al., Z. Phys. C 41 (1989) 567.

[6] L.A. Ahrens et al., Phys. Rev. D 41 (1990) 3297 .

[7] CHARM II Collab., Calibration and Performance of the CHARM-II Detector, in preparation.

[8] R. Marshall, Z. Phys. C 43 (1989) 607. 
[9] A.A. Bel'kov adn B.Z. Kopeliovich, Yad. Fiz. 46 (1987) 874.

[10] D.Yu. Bardin and V.A. Dokuchaeva, Nucl. Phys. B 246 (1984) 221;

Preprint JINR E2-86-260 (1986).

D.Yu. Bardin, NUFITTER, a program to calculate electroweak radiative correction for neutrino-electron scattering.
[11] M. Consoli and W. Hollik, in: G. Altarelli et al. (edts), Report CERN 89-08 (1989) 7.

[12] L3 Collab., B. Adeva et al., Z. Phys. C 51 (1991) 179.

[13] ALEPH Collab., D. Decamp et al., CERNPPE 91-105 (1991), subm. to Z. Phys. C. 

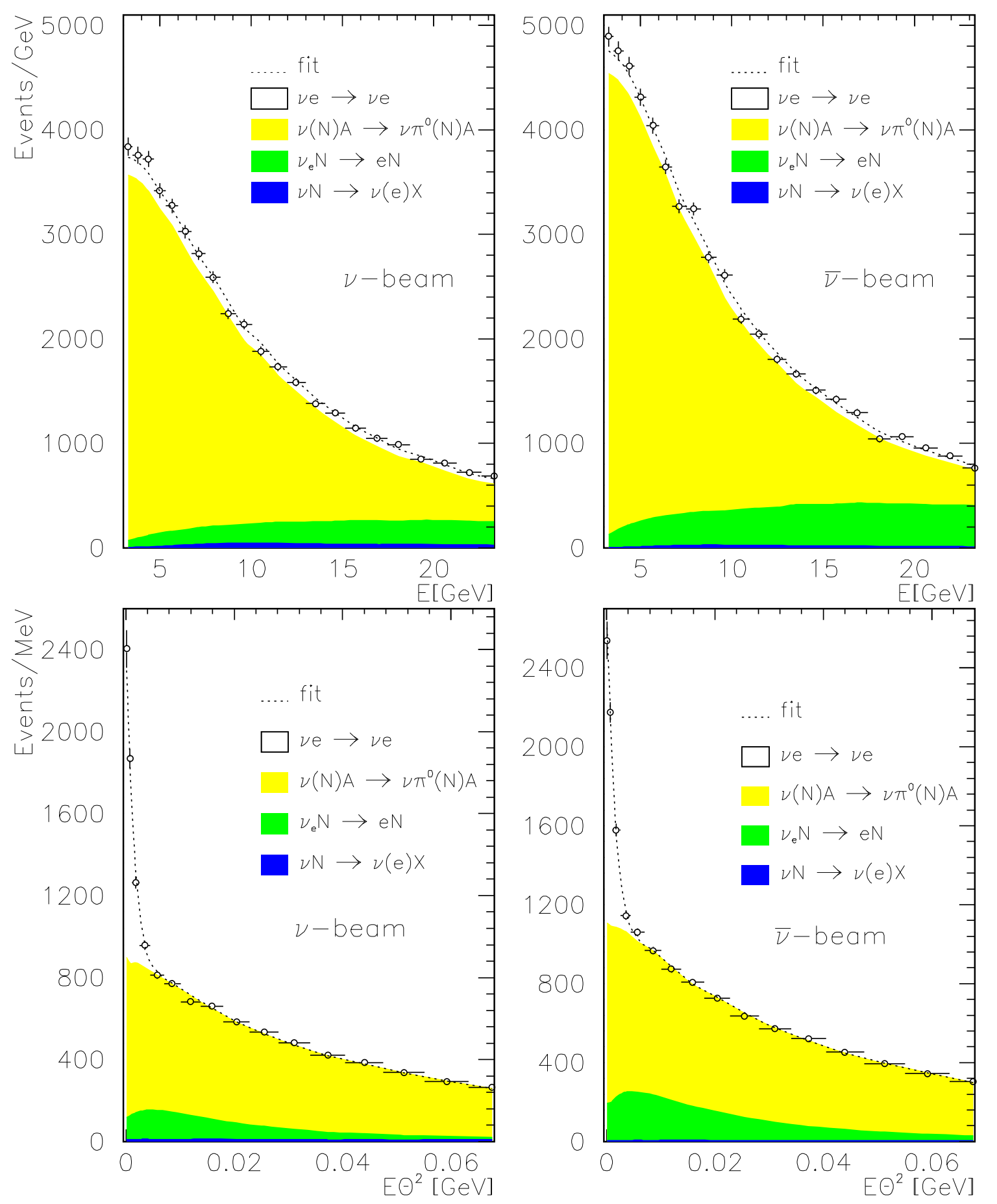

Figure 1: Experimental data and the result of the best fit: data are shown as circles and the fit results are displayed as a dashed line. Only the projections in $E_{e}$ and $E_{e} \theta_{e}^{2}$ of the 2-dim. distributions are shown. The different background components are added on top of each other. 


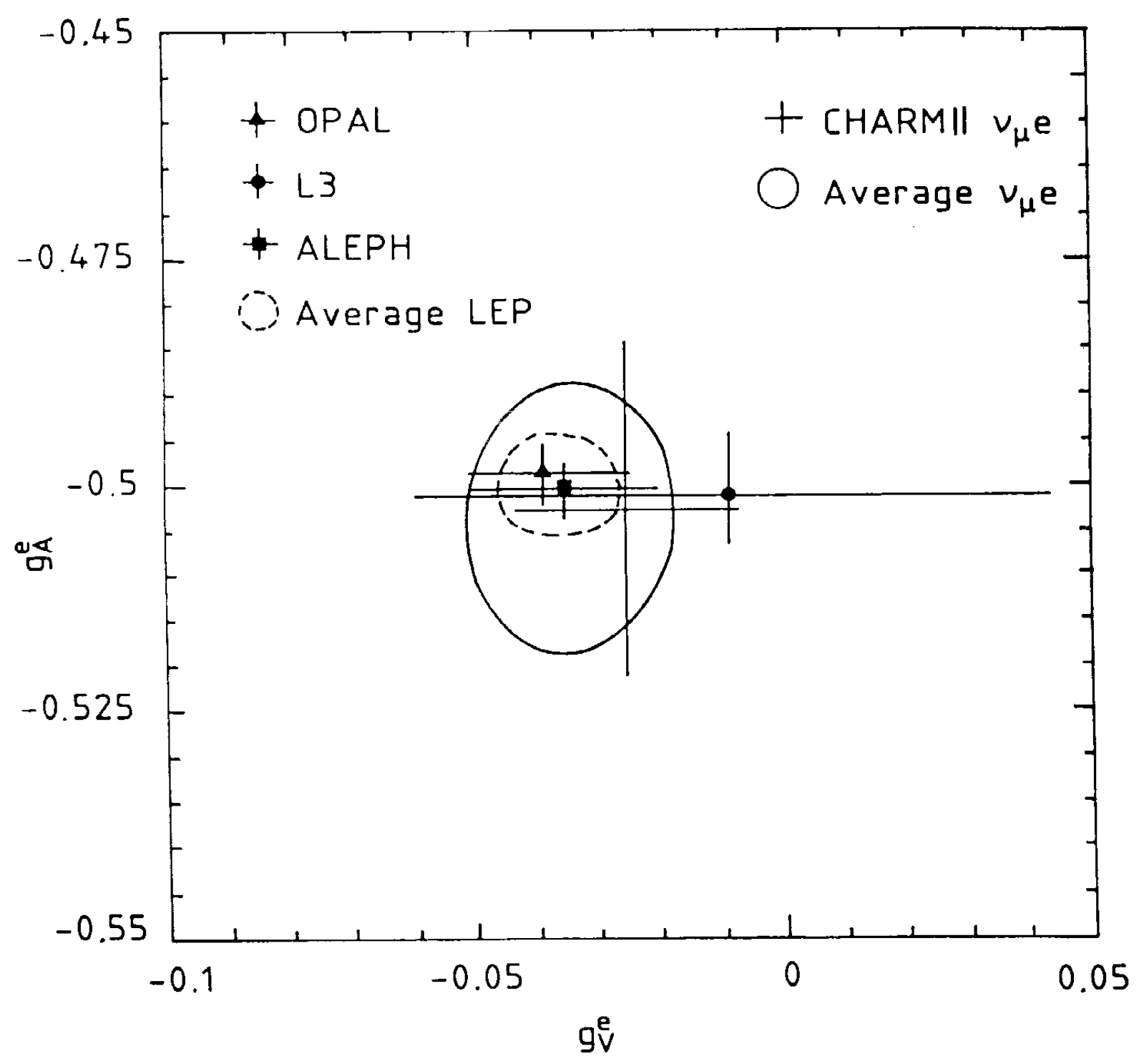

Figure 2: Comparison of results from neutrino-electron scattering and from $e^{+} e^{-} \rightarrow e^{+} e^{-}$annihilation at the $Z^{0}$ pole in the $g_{V}-g_{A}$-plane. The crosses show different experimental data points [1, 12, 13] and the contours (their projections correspond to one standard deviation) the averages obtained for the two different channels. 